\title{
Do some long COVID patients suffer from ME/CFS?
}

Chronic fatigue syndrome (CFS), also called myalgic encephalomyelitis (ME) and ME/CFS is characterized by rich symptomatology and relatively high variance of severity with about a quarter of patients being housebound or bedbound at some point in their illness [1]. There is a lack of unifying hypotheses on ME/CFS trigger/s; however, a role of vi ral pathogens solely or in combination with other stressors has been proposed [2]. Nevertheless, no specific pathogen triggering ME/CFS has, yet, been identified, although several group candidates have been proposed including viruses, bacteria, and parasites [2]. Seventy-seven percent of a cohort of $837 \mathrm{ME} / \mathrm{CFS}$ patients reported symptoms of acute infection (fever, upper respiratory tract infection, flu-like illness, or gastroenteritis) that occurred before the onset of $\mathrm{ME} / \mathrm{CFS}$ symptoms [3]. As a result, we speculate that at least some ME/CFS onsets might also be correlated in time with an unidentified infection.

It was reported that during the 1918 influenza pandemic, approximately $40 \%$ of patients developed chronic symptoms after the acute phase of the infection, including fatigue, lethargy, and difficulty concentrating; symptoms that worsened after exercise $[4,5]$. After the $\mathrm{H} 1 \mathrm{~N} 1$ pandemic in 2009, an increased incidence of $\mathrm{ME} / \mathrm{CFS}$ diagnoses was noted in Norway [6]. Based on the analysis of 233 cases of severe acute respiratory syndrome (SARS) survivors, it was noted that $27.1 \%$ fulfilled the Fukuda criteria for CFS $[7,8]$.

Several authors have noted an overlap between some long COVID patients and ME/CFS both in terms of symptomatology and the mechanism underlying it. Wong and Weitzer conducted a systemic review and comparison of the clinical presentation and symptomatology of those 2 entities. Three cardinal symptoms of ME/CFS, namely fatigue, reduced daily activity, and post-exertional malaise were reported in many analyzed studies examining long COVID patients [9]. Fatigue was the most reported symptom in studies of long COVID patients, which occurred in 13 of 21 analyzed studies. The symptoms that seem to be more unique to long COVID are olfactory and gustatory dysfunction and rash [9]. Nevertheless, in only three studies on long COVID analyzed in this review, patients' symptoms were tracked for six months or more [9]. Freitag et al. [10] examined 42 patients with a mostly mild acute phase of SARS-CoV-2 infection. On re-examination, after 6 months, it was noted that circa half of long COVID patients fulfill criteria for ME/CFS [10].

Persistent symptoms in post-viral disorders as in ME/CFS caused by a virus and in long COVID patients could be caused by a cascade response to a viral trigger. Paul et al. [11] suggested redox imbalance, systemic inflammation and neuroinflammation, an impaired ability to generate adenosine triphosphate, and a general hypometabolic state as factors underlying the pathophysiology of those two entities. Wostyn postulated the hypothesis that the glymphatic-lymphatic system might be disturbed in both long COVID and $\mathrm{ME} / \mathrm{CFS}$ patients and that cerebrospinal fluid drainage might serve as a long COVID treatment [12]. Another hypothesis considers a decrease of the pulsatile mode of the pituitary gland's secretion and a "vicious circle" that involves chronic inflammation, oxidative and nitrosative stress, and an underactive thyroid hormone [13].

Corresponding author: Paweł Zalewski, Department of Exercise Physiology and Functional Anatomy, Ludwik Rydygier Collegium Medicum in Bydgoszcz Nicolaus Copernicus University in Torun, Poland, e-mail: p.zalewski@cm.umk.pl

Medical Research Journal 2021; Volume 6, Number 4, 279-280, 10.5603/MRJ.a2021.0049, Copyright (C) 2021 Via Medica, ISSN 2451-2591, e-ISSN 2451-4101

This article is available in open access under Creative Common Attribution-Non-Commercial-No Derivatives 4.0 International (CC BY-NC-ND 4.0) license, allowing to download articles and share them with others as long as they credit the authors and the publisher, but without permission to change them in any way or use them commercially. 
Several authors have noted vascular dysfunction in COVID-19 pathogenesis [14-16], which might overlap with the endothelial dysfunction reported in ME/CFS patients $[17,18]$. In addition, disturbances in the autonomic nervous system, one of the hallmark symptoms of many ME/CFS patients [19], have been suggested as part of long COVID pathomechanism [20].

Given the relatively easy access to huge sample sizes of patients in the acute phase of COVID infection, more studies on large cohorts of patients are needed and achievable. It seems important to assess the dynamics of symptom quality and severity of COVID patients in a longitudinal manner. Risk factors for long COVID and its underlying biological mechanisms should be analyzed. Results of such studies might facilitate the development of preventive and therapeutic approaches for long COVID. Despite decades of research, there is neither a unifying hypothesis on ME/CFS pathogenesis nor biomarker nor effective methods that might lead to a cure for ME/CFS patients. If the hypothetical overlap in the mechanism underlying some subgroups of patients with long COVID and ME/CFS would be confirmed, then the development of therapeutic strategies for long COVID might have application and be beneficial for some ME/CFS patients.

\section{Conflict of interest: None.}

Funding: None.

\section{References}

1. Institute of Medicine (U.S.) Committee on the Diagnostic Criteria for Myalgic Encephalomyelitis/Chronic Fatigue Syndrome. Beyond myalgic encehalomyelitis/chronic fatigue syndrome: redefining an illness. National Academies Press, Washington DC 2015.

2. Friedman $\mathrm{KJ}$, Murovska M, Pheby DFH, et al. Our evolving understanding of ME/CFS. Medicina (Kaunas). 2021; 57(3): 200, doi 10.3390/medicina57030200, indexed in Pubmed: 33652622

3. Naess H, Sundal E, Myhr KM, et al. Postinfectious and chronic fatigue syndromes: clinical experience from a tertiary-referral centre in Norway In Vivo. 2010; 24(2): 185-188, indexed in Pubmed: 20363992.

4. Briggs NC, Levine PH. A comparative review of systemic and neurological symptomatology in 12 outbreaks collectively described as chronic fatigue syndrome, epidemic neuromyasthenia, and myalgic encephalomyelitis. Clin Infect Dis. 1994; 18(Suppl 1): S32-S42, doi: 10.1093/clinids/18.supplement_1.s32, indexed in Pubmed: 8148451.
5. Islam M, Cotler J, Jason L. Post-viral fatigue and COVID-19: lessons from past epidemics. Fatigue: Biomedicine, Health \& Behavior. 2020; 8(2): 61-69, doi: 10.1080/21641846.2020.1778227.

6. Magnus P, Gunnes N, Tveito K, et al. Chronic fatigue syndrome/myalgic encephalomyelitis (CFS/ME) is associated with pandemic influenza infection, but not with an adjuvanted pandemic influenza vaccine. Vaccine. 2015; 33(46): 6173-6177, doi: 10.1016/j.vaccine.2015.10.018, indexed in Pubmed: 26475444.

7. Lam MHB, Wing YK, Yu MWM, et al. Mental morbidities and chronic fatigue in severe acute respiratory syndrome survivors: longterm follow-up. Arch Intern Med. 2009; 169(22): 2142-2147, doi: 10.1001/archinternmed.2009.384, indexed in Pubmed: 20008700.

8. Fukuda K, Straus SE, Hickie I, et al. The chronic fatigue syndrome: a comprehensive approach to its definition and study. International Chronic Fatigue Syndrome Study Group. Ann Intern Med. 1994; 121(12): 953-959, doi: 10.7326/0003-4819-121-12-199412150-00009, indexed in Pubmed: 7978722.

9. Wong TL, Weitzer DJ. Long COVID and myalgic encephalomyelitis/chronic fatigue syndrome (ME/CFS) - a systemic review and comparison of clinical presentation and symptomatology. Medicina (Kaunas). 2021; 57(5): 418, doi: 10.3390/medicina57050418, indexed in Pubmed: 33925784

10. Kedor C, Freitag H, Meyer-Arndt L, et al. Chronic COVID-19 syndrome and chronic fatigue syndrome (ME/CFS) following the first pandemic wave in Germany - a first analysis of a prospective observational study. medRxiv. 2021, doi: 10.1101/2021.02.06.21249256.

11. Paul BD, Lemle MD, Komaroff AL, et al. Redox imbalance links COVID-19 and myalgic encephalomyelitis/chronic fatigue syndrome. Proc Natl Acad Sci U S A. 2021; 118(34), doi: 10.1073/pnas.2024358118, indexed in Pubmed: 34400495.

12. Wostyn P. COVID-19 and chronic fatigue syndrome: is the worst yet to come? Med Hypotheses. 2021; 146: 110469, doi: 10.1016/j. mehy.2020.110469, indexed in Pubmed: 33401106.

13. Stanculescu D, Larsson L, Bergquist J. Hypothesis: mechanisms that prevent recovery in prolonged ICU patients also underlie myalgic encephalomyelitis/chronic fatigue syndrome (ME/CFS). Front Med (Lausanne). 2021; 8: 628029, doi: 10.3389/fmed.2021.628029, indexed in Pubmed: 33585528.

14. Fan BE, Cheung C. Post COVID-19 arterial thromboembolism: a clear and present danger. Semin Thromb Hemost. 2021 [Epub ahead of print], doi: 10.1055/s-0041-1728717, indexed in Pubmed: 33860515.

15. Wazny V, Siau A, Wu KX, et al. Vascular underpinning of COVID-19. Open Biol. 2020; 10(8): 200208, doi: 10.1098/rsob.200208, indexed in Pubmed: 32847471

16. Rovas A, Osiaevi I, Buscher K, et al. Microvascular dysfunction in COVID-19: the MYSTIC study. Angiogenesis. 2021; 24(1): 145-157. doi: 10.1007/s10456-020-09753-7, indexed in Pubmed: 33058027.

17. Scherbakov N, Szklarski M, Hartwig J, et al. Peripheral endothelial dysfunction in myalgic encephalomyelitis/chronic fatigue syndrome. ESC Heart Fail. 2020; 7(3): 1064-1071, doi: 10.1002/ehf2.12633, indexed in Pubmed: 32154656

18. Blauensteiner J, Bertinat $R$, León LE, et al. Altered endothelial dysfunction-related miRs in plasma from ME/CFS patients. Sci Rep. 2021; 11(1): 10604, doi: 10.1038/s41598-021-89834-9, indexed in Pubmed: 34011981

19. Nelson MJ, Bahl JS, Buckley JD, et al. Evidence of altered cardiac autonomic regulation in myalgic encephalomyelitis/chronic fatigue syndrome: a systematic review and meta-analysis. Medicine (Baltimore). 2019; 98(43): e17600, doi: 10.1097/MD.0000000000017600, indexed in Pubmed: 31651868

20. Barizien N, Le Guen M, Russel S, et al. Clinical characterization of dysautonomia in long COVID-19 patients. Sci Rep. 2021; 11(1): 14042, doi: 10.1038/s41598-021-93546-5, indexed in Pubmed: 34234251. 